\title{
Application of Time-varying Acceleration Coefficients PSO to Face Pose Estimation
}

\author{
Yudong ZHANG ${ }^{1}$, Shuihua WANG ${ }^{1}$, Genlin $\mathrm{JI}^{1}$ \\ ${ }^{1}$ School of Computer Science and Technology, Nanjing Normal University, Nanjing, Jiangsu \\ 210023, China \\ Email: zhangyudong@njnu.edu.cn
}

\begin{abstract}
Keywords:Face pose estimation; particle swarm optimization; time-varying acceleration coefficients; genetic algorithm; noise-free.
\end{abstract}

\begin{abstract}
This study focuses on the problem of human face pose estimation based on single image. Traditional methods for 2D-3D feature based pose estimation problem require two inputs, and they cannot work well due to lack of correspondences of input images. We transfer the problem into an optimization problem via six-point template, and solve the problem by time-varying acceleration coefficients particle swarm optimization (TVAC-PSO). Experiments on 40 different poses demonstrate that the TVAC-PSO is superior to either GA or PSO in terms of accuracy.
\end{abstract}

\section{Introduction}

In computer vision, a typical task is to identify specific objects in an image and to determine each object's position and orientation relative to some coordinate system[1]. The combination of position and orientation is referred to as the "pose" of an object[2]. This concept is sometimes used only to describe the orientation[3].

Pose estimation can be considered as the process of determining the object reference frame w.r.t. the camera reference frame, giving some certain measurements of the object in both frames[4]. The image data from which the pose of an object is determined can be either a single image, a stereo image pair, or an image sequence[5]. The objects can be rather general, including a living being or body parts, e.g., the human face[6]. In this study, we center in the face pose estimation.

Researchers have proposed various methods to solve the face pose estimation problem. At present those methods fall into two categories[7-9]: (i) Appearance-based approach. The oriented face image is regarded as the whole input to estimate the pose, and (ii) Feature-based approach. Several pairs of corresponding points, lines or curves are employed to approximate solution by iteration[10].The most classical algorithm is the Perspective N-Point problem [11], where $\mathrm{N}$ is the number of points used and often takes 3,4 , or 5 or more.The former approach may have an accurate result for certain people's face pose, but it needs complicated calculations and is sensitive to different types of faces[12]. Therefore, we focus on the second category. The methods in the second category use the algebraic nature of the problem. They formulate it as an optimization problem.

The problem rises as how to solve the optimization efficiently and robustly. Traditional gradient-based methods have accuracy in estimating the translational parameters but give large errors in estimating the rotational parameters. Statisticians have already proven that when noise level exceeds a given threshold or the number of feature points is below a given threshold, the errors in pose estimation by the gradient-based methods will increase to a high value dramatically.

Sattar et al. used genetic algorithm (GA) to determine the parameters from the knowledge of a given set of points [13]. Zhang et al. proposed a particle swarm optimization (PSO) to get the orientation parameters of two faces [5]. Unfortunately, the estimation errors of both GA and PSOare a bit large. In last decade, scholars have proposed numerous variants of PSO, such as Fitness-scaling PSO (FSPSO) [14], Adaptive Chaotic PSO (ACPSO) [15], Bi-Velocity discrete 
PSO (BVDPSO) [16], Time-varying Acceleration Coefficients PSO (TVAC-PSO) [17], Chaotic Catfish PSO (CCPSO) [18], Restarted Simulated Annealing PSO (RSAPSO) [19], Chaotic Immune PSO (CIPSO) [20], etc. Among those variants, the TVAC-PSO is competitive with other state-of-the-art variants, and is applied successfully in many fields [21-23]. Hence, we decide to employ TVAC-PSO to solve the optimization problem.

\section{Problem Model}

The transform between two frames is formulated by the combination of rotation matrix $\mathrm{R}$ and translation matrix $\mathrm{T}$. The approaches of estimating translation matrix $\mathrm{T}$ were discussed in many literatures; therefore, this paper mainly focuses on the estimation of rotation matrix R. The relationship between object space and image space are given as follow

$$
\left[\begin{array}{l}
x^{\prime} \\
y^{\prime} \\
z^{\prime}
\end{array}\right]=R\left[\begin{array}{l}
x \\
y \\
z
\end{array}\right]+T,\left[\begin{array}{l}
u \\
v
\end{array}\right]=\left[\begin{array}{l}
f x^{\prime} / z^{\prime} \\
f y^{\prime} / z^{\prime}
\end{array}\right]
$$

Here $f$ denotes for the focal length of the camera defined beforehand. $(x, y, z)^{\mathrm{T}},\left(x^{\prime}, y^{\prime}, z^{\prime}\right)^{\mathrm{T}}$, and $(u, v)^{\mathrm{T}}$ denote for the feature points in the object space, image space and the image plane, respectively. The rotation matrix $\boldsymbol{R}$ can be decomposed to three sub-rotation matrices as $R=R_{x}\left(\theta_{x}\right) R_{y}\left(\theta_{y}\right) R_{z}\left(\theta_{z}\right)$, where $\theta_{x}, \theta_{y}, \theta_{z}$ are three rotational angles along the $x$-axis, $y$-axis, $z$-axis, respectively. $R_{x}\left(\theta_{x}\right), R_{y}\left(\theta_{y}\right), R_{z}\left(\theta_{z}\right)$ are the corresponding rotation matrices.

$$
R_{x}\left(\theta_{x}\right)=\left(\begin{array}{ccc}
1 & 0 & 0 \\
0 & \cos \left(\theta_{x}\right) & -\sin \left(\theta_{x}\right) \\
0 & \sin \left(\theta_{x}\right) & \cos \left(\theta_{x}\right)
\end{array}\right), R_{y}\left(\theta_{y}\right)=\left(\begin{array}{ccc}
\cos \left(\theta_{y}\right) & 0 & \sin \left(\theta_{y}\right) \\
0 & 1 & 0 \\
-\sin \left(\theta_{y}\right) & 0 & \cos \left(\theta_{y}\right)
\end{array}\right), R_{z}\left(\theta_{z}\right)=\left(\begin{array}{ccc}
\cos \left(\theta_{z}\right) & -\sin \left(\theta_{z}\right) & 0 \\
\sin \left(\theta_{z}\right) & \cos \left(\theta_{z}\right) & 0 \\
0 & 0 & 1
\end{array}\right)
$$

Thus, the pose estimation is described as following problem: Given a face image of arbitrary pose, how to get the three rotation angles of the face image w.r.t. his (or her) own front face image[24].

In this study, we choose 6 points as the template points, which contain two outer eye corners, two outer mouth corners, nose tip and chin tip[25]. The automatic selection method is out of scope of this paper. They are marked in sequence as point $1,2, \ldots$, till 6 . The origin is set as located at the nose tip position. The 6 points pi $(\mathrm{i}=1,2, \ldots, 6)$ in the 3D coordinates are expressed as follows.

$$
\left(p_{1}, p_{2}, \ldots, p_{6}\right)=\left[\begin{array}{cccccc}
-x_{e} & x_{e} & 0 & 0 & -x_{m} & x_{m} \\
y_{e} & y_{e} & -y_{c} & 0 & -y_{m} & -y_{m} \\
z_{e} & z_{e} & z_{c} & 0 & z_{m} & z_{m}
\end{array}\right]
$$

It should be noted that the $x_{e}, x_{m}, y_{e}, y_{c}, y_{m}$ are all greater than 0 and $z_{e}, z_{c}, z_{m}$ are all less than 0 . Then, suppose those 6 points are projected into the image plane, we get 6 image points $q^{\prime}{ }_{i}$ $(i=1,2, \ldots, 6)$.

$$
q_{i 1}^{\prime}=f \frac{r_{1} x_{i}+t_{1}}{r_{3} x_{i}+t_{3}}, q_{i 2}^{\prime}=f \frac{r_{2} x_{i}+t_{2}}{r_{3} x_{i}+t_{3}}, i=1,2, \ldots 6
$$

Here, $r_{1}, r_{2}$, and $r_{3}$ are the row vectors of rotation matrix $\boldsymbol{R}, t_{1}, t_{2}$, and $t_{3}$ are the row component of translation matrix $\boldsymbol{T}$. We set $\boldsymbol{T}$ to 0 for simple, since only rotational angles are considered in this paper.

On the basis of this 6-point template, we employed the distance function $D=\sum_{i=1}^{6}\left|q_{i}-q_{i}\right|$ as the objective function, where qi denotes the feature points labeled manually. Our objective is to find the optimum values of the three rotational parameters aiming at minimizing objective function D. 


\section{Optimization Algorithm}

PSO performs searching via a swarm of particles that updates from iteration to iteration[2628]. To seek the optimal solution, each particle moves in the direction to its previously best (pbest) position and the global best (gbest) position in the swarm[29-31].The velocity $\mathrm{V}$ of particles is updated by

$$
V_{i}(t+1)=\omega V_{i}(t)+c_{1} r_{1}\left(\text { pbest }(i, t)-P_{i}(t)\right)+c_{2} r_{2}\left(g \text { gest }(t)-P_{i}(t)\right)
$$

The inertia weight $\omega$ is used to balance the global exploration and local exploitation. The r1 and r2 are uniformly distributed random variables within range $[0,1]$. The c1 and c2 are positive constant parameters called “acceleration coefficients”[32-34].

The Time Varying Acceleration Coefficients (TVAC) technique is introduced, and we call the combined algorithm of both PSO and TVAC as TVAC-PSO. It can enhance the global search ability in the early stage, and encourage the local search ability of particles at the end of the search. In order to achieve this goal, TVAC-PSO gives more weight on cognitive component and less weight on social component at the former stage, and gives less weight on cognitive component and more weight on social component in the latter stage. Mathematically, TVAC-PSO tunes c1 and c2 as

$$
c_{1}=\left(c_{1 f}-c_{1 i}\right) * \frac{t}{M A X_{-} \text {Iter }}+c_{1 i}, c_{2}=\left(c_{2 f}-c_{2 i}\right) * \frac{t}{M A X_{-} \text {Iter }}+c_{2 i}
$$

where $c_{1 \mathrm{i}}$ and $c_{1 \mathrm{f}}$ represents the initial and final value of $c_{1}$, respectively. $c_{2 \mathrm{i}}$ and $c_{2 \mathrm{f}}$ represents the initial and final value of $c_{2}$, respectively.

\section{Experiments}

The template subject with 40 poses were photographed and taken as test images, of which the three rotational angles are uniformly distributed within the range $[0,180]$ in unit of degree. GA[13] and PSO[5] are chosen as the comparison basis.

First, we estimate three angles of rotationat the condition without noises. The errors distributions of GA, PSO, and TVAC-PSO over all 40 poses are shown in Figure 1. The central red mark represents the median, the edges of the box are the 25th and 75th percentiles, and the whiskers extend to the most extreme data points. Outliers are plot individually if exists. The mean and standard deviation (Std.) of the error values are listed in Table 1.

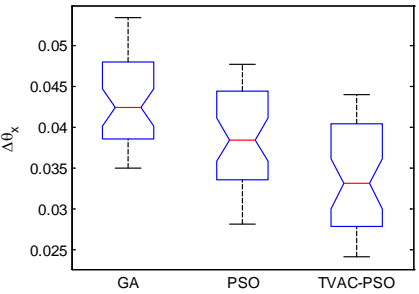

(a) $\Delta \theta_{x}$

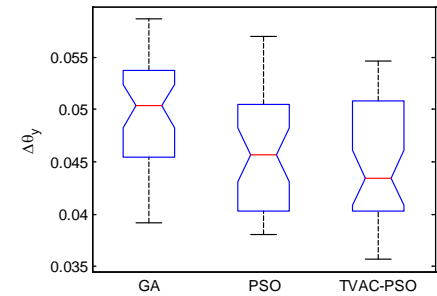

(b) $\Delta \theta_{y}$

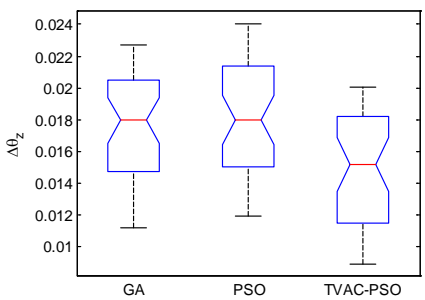

(c) $\Delta \theta_{z}$

Figure 1Distributions of errors on three angles of rotation.

Table 1 Statistical Description on errors of three angles of rotation

\begin{tabular}{lllllll}
\hline Error & GA[13] & \multicolumn{2}{c}{ PSO[5] } & \multicolumn{2}{c}{ TVAC-PSO } \\
& Mean & Std. & Mean & Std. & Mean & Std. \\
\hline$\Delta \boldsymbol{\theta}_{\boldsymbol{x}}$ & 0.0445 & 0.0058 & 0.0384 & 0.0069 & 0.0347 & 0.0060 \\
$\Delta \boldsymbol{\theta}_{\boldsymbol{y}}$ & 0.0484 & 0.0065 & 0.0482 & 0.0063 & 0.0451 & 0.0057 \\
$\Delta \boldsymbol{\theta}_{\boldsymbol{z}}$ & 0.0168 & 0.0039 & 0.0179 & 0.0034 & 0.0151 & 0.0034 \\
\hline
\end{tabular}

Noise-free are impractical; hence, the Gaussian noises are added to the coordinates of the 6 feature points. The range of variances of the noise is set from 0.01 to 0.05 with increasing step 
of 0.01. Afterwards, the mean error of different algorithms over 40 samples are calculated, and plotted against the variances of noise. The curves are shown in Figure 2.

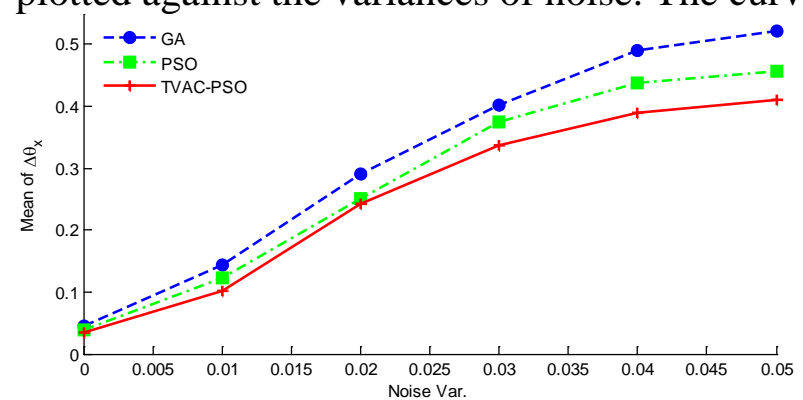

(a) $\Delta \theta_{x}$

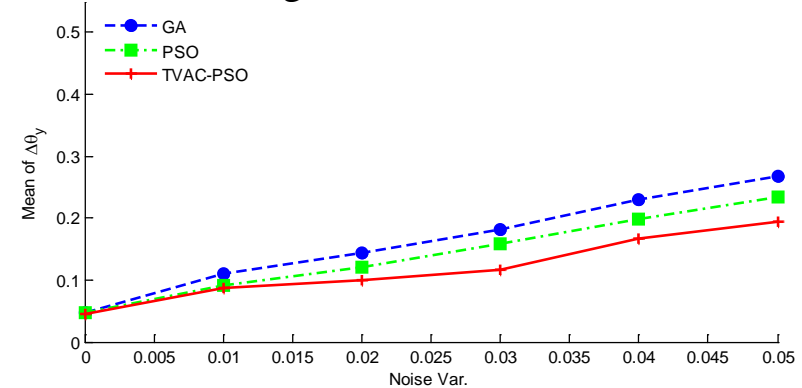

(b) $\Delta \theta_{y}$

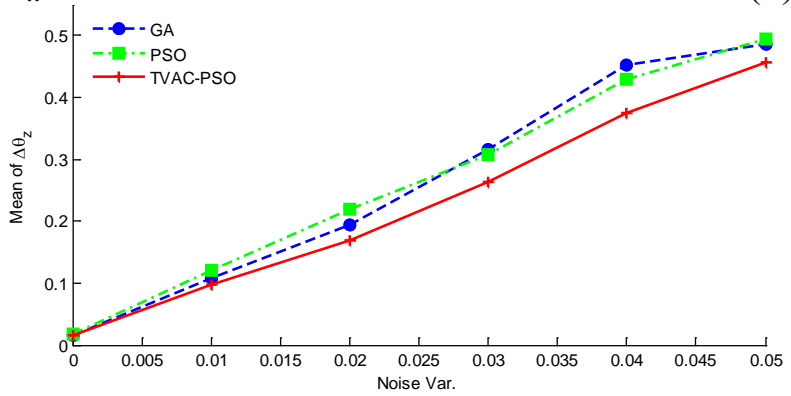

(c) $\Delta \theta_{z}$

Figure 2Mean of estimation errors of three rotational anglesagainst variance of noise

\section{Discussions and Conclusions}

It is easily observed from results in Table 1 that: for $\Delta \theta \mathrm{x}$, the mean error of GA is the highest of 0.0445 , PSO reduces it to only 0.0384 , and TVAC-PSO obtains the least error of 0.0347 . For $\Delta \theta y$, GA again performs worst with mean error of 0.0484 , PSO ranks second of 0.0482 , and the TVAC-PSO of only 0.0451 . For $\Delta \theta z$, PSO performs worst with mean error of 0.0179 , followed by GA of 0.0168 , and the proposed TVAC-PSO has the best result of merely 0.0151 . Furthermore, the difference of the Std. between TVAC-PSO and GA/PSO is not statistically significant. This is our future research direction. In addition, the proposed TVACPSOalgorithm excels both GA and PSO in noise-free cases. The three rotational parameters estimated by TVAC-PSO are quite close to their corresponding realistic values.

The curves in Figure 2 suggests that estimation errors are acceptable $(<0.55$ degree) under the condition that the noise variance is not larger than 0.05 . Generally, most of practical conditions meet this constrain. Therefore, the proposed method is practical. Besides, the curve of the proposed TVAC-PSO increases not as sharply as GA and PSO method, which indicates that the TVAC-PSO is less sensitive to noises. In addition, the rotational angle along y-axis has a tendency of increases relatively slowly compared to the angle along $\mathrm{x}$ - and $\mathrm{z}$-axis. This suggests us the estimation of $\Delta \theta \mathrm{y}$ may be more robust than $\Delta \theta \mathrm{x}$ and $\Delta \theta \mathrm{z}$. The reason may lie in the image acquiring method of our experiment. We will try to increase the number of subjects and the number of poses in the experiment design.

The contribution of this paper focuses on following aspects: (i) We apply the TVAC-PSO to the field of face pose estimation; (ii) We prove that the TVAC-PSO estimate in average more closely to realistic values in estimating three rotational angles at either noise-free or noisy conditions. (iii) We find TVAC-PSO did not improve the standard deviation compared to GA and PSO.

\section{References}

1. Saquib Sarfraz, M. and O. Hellwich, Probabilistic learning for fully automatic face recognition across pose. Image and Vision Computing, 2010. 28(5): p. 744-753. 
2. Li, D.Q. and W. Pedrycz, A central profile-based 3D face pose estimation. Pattern Recognition, 2014. 47(2): p. 525-534.

3. Qiao, X., et al., Tensor-based subspace learning and its applications in multi-pose face synthesis. Neurocomputing, 2010. 73(13-15): p. 2727-2736.

4. Van der Linde, I. and T. Watson, A combinatorial study of pose effects in unfamiliar face recognition. Vision Research, 2010. 50(5): p. 522-533.

5. Zhang, Y., et al. Face Orientation Estimation by Particle Swarm Optimization. in Information Science and Engineering (ISISE), 2009 Second International Symposium on. 2009. p. 388-391.

6. Voelkl, E., Noise in off-axis type holograms including reconstruction and CCD camera parameters. Ultramicroscopy, 2010. 110(3): p. 199-210.

7. Jimenez, P., et al., Face pose estimation with automatic $3 D$ model creation in challenging scenarios. Image and Vision Computing, 2012. 30(9): p. 589-602.

8. Niese, R., et al., Accurate, Fast and Robust Realtime Face Pose Estimation using Kinect Camera. 2013 Ieee International Conference on Systems, Man, and Cybernetics (Smc 2013), 2013: p. 487-490.

9. Iuspa, L. and E. Ruocco, Optimum topological design of simply supported composite stiffened panels via genetic algorithms. Computers \& Structures, 2008. 86(17-18): p. 17181737.

10. Zhang, Y.D. and L.N. Wu, Segment-based coding of color images. Science in China Series F-Information Sciences, 2009. 52(6): p. 914-925.

11. Kollreider, K., H. Fronthaler, and J. Bigun, Non-intrusive liveness detection by face images. Image and Vision Computing, 2009. 27(3): p. 233-244.

12. Hazar, M., H. Mohamed, and B. Hanene, Real-Time Face Pose Estimation in Challenging Environments, in Advanced Concepts for Intelligent Vision Systems, Acivs 2013, J. BlancTalon, et al., Editors. 2013. p. 114-125.

13. Sattar, A. and R. Seguier. GGA-AAM: Novel heuristic method of gradient driven Genetic Algorithm for Active Appearance Models. in Digital Information Management, 2008. ICDIM 2008. Third International Conference on. 2008. p. 45-50.

14. Zhang, Y., L. Wu, and S. Wang, UCAV Path Planning by Fitness-Scaling Adaptive Chaotic Particle Swarm Optimization. Mathematical Problems in Engineering, 2013. 2013: p. 9.

15. Zhang, Y. and L. Wu, Crop Classification by forward neural network with adaptive chaotic particle swarm optimization. Sensors, 2011. 11(5): p. 4721-4743.

16. Shen, M., et al., Bi-Velocity Discrete Particle Swarm Optimization and Its Application to Multicast Routing Problem in Communication Networks. Ieee Transactions on Industrial Electronics, 2014. 61(12): p. 7141-7151.

17. Abdullah, M.N., et al., Modified Particle Swarm Optimization with Time Varying Acceleration Coefficients for Economic Load Dispatch with Generator Constraints. Journal of Electrical Engineering \& Technology, 2014. 9(1): p. 15-26.

18. Chuang, L.-Y., S.-W. Tsai, and C.-H. Yang, Chaotic catfish particle swarm optimization for solving global numerical optimization problems. Applied Mathematics and Computation, 2011. 217(16): p. 6900-6916.

19. Zhang, Y., et al., Binary Structuring Elements Decomposition Based on an Improved Recursive Dilation-Union Model and RSAPSO Method. Mathematical Problems in Engineering, 2014. 2014: p. 12.

20. Zhang, Y., et al., Find multi-objective paths in stochastic networks via chaotic immune PSO. Expert Systems with Applications, 2010. 37(3): p. 1911-1919.

21. Chih, M.C., et al., Particle swarm optimization with time-varying acceleration coefficients for the multidimensional knapsack problem. Applied Mathematical Modelling, 2014. 38(4): p. 1338-1350. 
22. Pookpunt, S. and W. Ongsakul, Optimal placement of wind turbines within wind farm using binary particle swarm optimization with time-varying acceleration coefficients. Renewable Energy, 2013. 55: p. 266-276.

23. Abedinia, O., et al., Solution of economic load dispatch problem via hybrid particle swarm optimization with time-varying acceleration coefficients and bacteria foraging algorithm techniques. International Transactions on Electrical Energy Systems, 2013. 23(8): p. 1504-1522.

24. Kim, W., et al., A Feasible Face Pose Estimation by Evaluating 3D Facial Feature Vectors from 2D Features, in 2013 15th International Conference on Advanced Communication Technology2013. p. 668-671.

25. Wu, B.F., et al., ACTIVE APPEARANCE MODEL ALGORITHM WITH K-NEAREST NEIGHBOR CLASSIFIER FOR FACE POSE ESTIMATION. Journal of Marine Science and Technology-Taiwan, 2014. 22(3): p. 285-294.

26. Zhang, Y., et al., Binary PSO with mutation operator for feature selection using decision tree applied to spam detection. Knowledge-Based Systems, 2014. 64(0): p. 22-31.

27. Samanta, C.K., et al., Hybrid swarm intelligence methods for energy management in hybrid electric vehicles. Electrical Systems in Transportation, IET, 2013. 3(1): p. 22-29.

28. Alam, S., et al., Research on particle swarm optimization based clustering: A systematic review of literature and techniques. Swarm and Evolutionary Computation, 2014. 17(0): p. 113.

29. Zhang, Y., S. Wang, and L. Wu, A Novel Method for Magnetic Resonance Brain Image Classification based on Adaptive Chaotic PSO. Progress in Electromagnetics Research, 2010. 109: p. 325-343.

30. Sathya, P.D. and R. Kayalvizhi, Amended bacterial foraging algorithm for multilevel thresholding of magnetic resonance brain images. Measurement, 2011. 44(10): p. 1828-1848.

31. Sakthivel, V.P., R. Bhuvaneswari, and S. Subramanian, Multi-objective parameter estimation of induction motor using particle swarm optimization. Engineering Applications of Artificial Intelligence, 2010. 23(3): p. 302-312.

32. Zhang, Y., et al., An MR Brain Images Classifier System via Particle Swarm Optimization and Kernel Support Vector Machine. The Scientific World Journal, 2013. 2013: p. 9.

33. Hosseinnezhad, V., et al., Species-based Quantum Particle Swarm Optimization for economic load dispatch. International Journal of Electrical Power \& Energy Systems, 2014. 63(0): p. 311-322.

34. Fahmy, A., T.M. Hassan, and H. Bassioni, Improving RCPSP solutions quality with Stacking Justification - Application with particle swarm optimization. Expert Systems with Applications, 2014. 41(13): p. 5870-5881. 